\title{
Free-Standing Microcrystals of a Fluorescent Organic Dye: Preparation and Spectroscopic Study
}

\author{
Mouhammad Abyan, Liliana Bîrlă, Franck Bertorelle, Fernanda Rodrigues, and Suzanne Fery-Forgues \\ Laboratoire des Interactions Moléculaires Réactivité Chimique et Photochimique, UMR CNRS 5623, Université Paul Sabatier, \\ 31062 Toulouse Cedex 9, France
}

Received 25 February 2005; Accepted 7 June 2005

Suspensions of microcrystals were obtained from an organic fluorescent dye, 4- $n$-octylamino-7-nitrobenz-2-oxa-1,3-diazole, by reprecipitation in water in the presence of various macromolecules: anionic or cationic PAMAM dendrimer, poly(acrylic acid, sodium salt), poly(acrylamide), and calf thymus DNA. The use of these additives allowed the size and shape of the microcrystals to be controlled. A study by UV/vis absorption and fluorescence spectroscopy revealed some common points, but also some interesting differences, in the optical behaviour of the five samples.

Copyright (c) 2006 Mouhammad Abyan et al. This is an open access article distributed under the Creative Commons Attribution License, which permits unrestricted use, distribution, and reproduction in any medium, provided the original work is properly cited.

\section{INTRODUCTION}

Organic nano- and microcrystals are defined as very tiny particles, the size of which does not exceed a hundred of nanometers or some micrometers, respectively. Their physicochemical properties considerably differ from those of individual molecules, due to the weak intermolecular interaction forces of the Van der Waals type that take place in the organic solid state [1]. On the other hand, their properties do not resemble those of bulk crystals, since they are closely related to the large proportion of surface molecules that characterizes nano- and microcrystals. Consequently, these small objects can be seen as an intermediate state, bridging the gap between single molecules and bulk materials. They give access to original applications in various fields of high technology, such as bioanalysis [2], photocatalysis [3], photonics, and microelectronics $[4,5]$. In the more traditional field of pharmaceutical sciences, nano- and microcrystals are also of high interest for improving the bioavailability of hydrophobic drugs $[6,7]$.

However, the ability to tailor the physical characteristics of organic microcrystals in view of a given application still represents an important challenge. Actually, due to the thermal instability of organic compounds, few methods of preparation can be used. Carrying out microcrystallization in spatially restricted environments, such as those afforded by microemulsions [8], polymer matrices [9], and sol-gel glasses $[10,11]$, is an efficient way to keep some control on the crystal size. This allows fundamental studies to be made on the crystal properties. However, free-standing microcrystals are probably better suited for subsequent applications, especially when the preparation method can be extended to a wider scale. In this respect, the reprecipitation method has shown to be an effective approach [4]. Based on a solvent exchange process at room temperature, this soft method consists in dissolving the organic compound in a good solvent. Then the concentrated organic solution is poured into a second solvent, for example, water, in which the organic compound is insoluble. As a result, the organic compound precipitates, and can eventually give microcrystals in suspension. In this basic framework, several parameters can be tuned with the aim to gain control on the microcrystal size. These parameters are, for example, the concentration of the injected solution and the temperature [12], the use of microwaves [13] or ultrasounds [14], the time left to the suspension for aging [15], and the addition of various substances in the reprecipitation medium [12, 16-18]. Very often, the substances used as additives are small molecules, such as surfactants, that get inserted into the crystal network and interfere with crystal growth.

In the last few years, our team has been using the reprecipitation method to produce microcrystals from an organic compound, namely, 4- $n$-octylamino-7-nitrobenz-2oxa-1,3-diazole (1). Dyes of this series have for long been known for their excellent optical properties, and are currently used as fluorescent probes to report on the polarity 


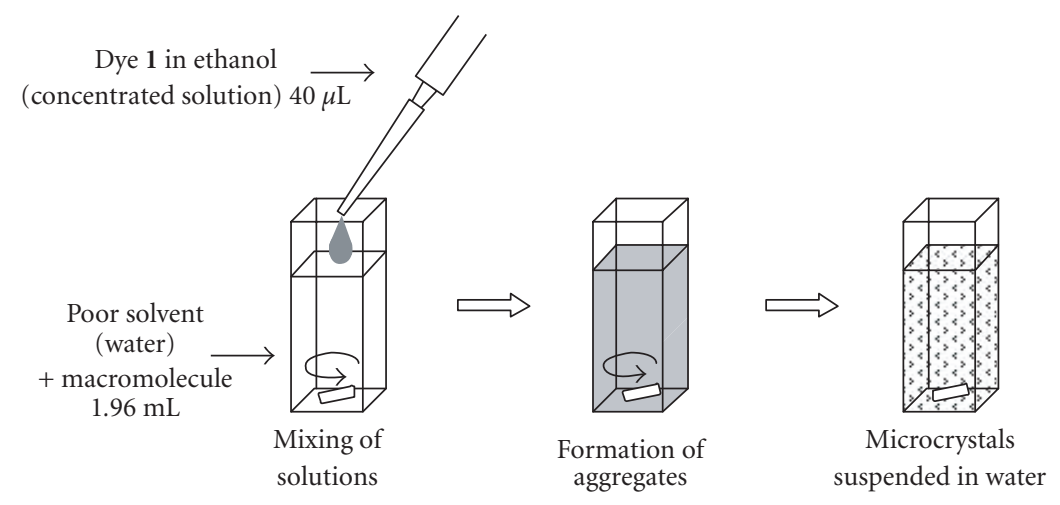

FIGURE 1: Schematic representation of the reprecipitation method.

and acidity of their environment $[19,20]$. The originality of our work is that various macromolecules, and not small molecules, were used as additives in the reprecipitation process. These macromolecules were starburst dendrimers of the poly(amidoamine) (PAMAM) type, provided with 64 carboxylate or ammonium terminal groups, synthetic linear polymers such as poly(acrylic acid, sodium salt) and neutral poly(acrylamide), and a natural polymer, calf thymus DNA. Each of them allowed to prepare, on a reproducible manner, suspensions of microcrystals that exhibit distinct morphological characteristics. The aim of the present paper is to give an overview of these recent investigations. We tried to point out the common points as well as the differences in spectroscopic behaviour for the various microcrystals obtained.

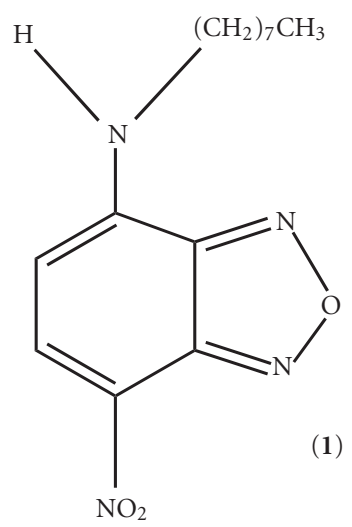

\section{RESULTS AND DISCUSSION}

\subsection{Effect of macromolecules upon the reprecipitation process}

In a typical experiment, dye $\mathbf{1}$ was dissolved in absolute ethanol to give a concentrated solution at $1 \times 10^{-3} \mathrm{M}$, then a small amount of this solution was rapidly injected into 49 times its volume of water. The concentration of dye in the water-ethanol medium was then $2 \times 10^{-5} \mathrm{M}$ that is much higher than the solubility threshold $\left(4 \times 10^{-7} \mathrm{M}\right)$ [21]. In these conditions, the yellow mixture lost its colour progressively, while compound 1 precipitates, giving red agglomerates of microcrystals on the vessel walls and on the stirrer.
This process, which was monitored by UV/vis spectroscopy, was completed within one hour. Its kinetics was studied in a previous work [22].

When reprecipitation was carried out in the presence of a macromolecule used as additive, the macromolecule was placed into water before the concentrated solution of dye was added (Figure 1). The influence of the macromolecule concentration was studied in every case, but most of the experiments were done with PAMAM G3.5 dendrimer at $4 \times 10^{-5} \mathrm{M}, \mathrm{G} 4$ dendrimer at $5 \times 10^{-5} \mathrm{M}$, and poly(acrylic acid) at $5.9 \times 10^{-4} \mathrm{M}$, or $5.9 \times 10^{-5} \mathrm{M}$. Poly(acrylamide) was used at a concentration of $4.1 \times 10^{-7}$ (that is, $3.2 \times 10^{-2} \mathrm{M}$ of terminal groups) or $4.1 \times 10^{-8} \mathrm{M}$. As for DNA, its concentration was set at $5 \times 10^{-5} \mathrm{M}$ bp to keep the viscosity of the medium rather low. The dye solution was then injected in the aqueous medium containing the macromolecule. The initial yellow solution progressively turned light pink. All ionic macromolecules accelerated drastically the reprecipitation process, the latter being, for example, completed 10 times faster in the presence of dendrimers than in pure water. With poly(acrylamide), the duration of the process was the same as in water alone, although the kinetics was different.

The acceleration of the reprecipitation process that is observed in most of the cases shows that the macromolecules do not behave like small additives, which generally induce a slowing down of the crystallization process. The first step of the reprecipitation process is the formation of aggregate from the dissolved molecules. This step was strongly accelerated for ionic dendrimers and poly(acrylic acid) [22, 23]. It was unchanged for poly(acrylamide) and DNA. Two hypotheses can be proposed to explain the effect of the additives observed in the first case. (i) The macromolecule affects the structuring of water, so the solvation of the dye molecules is reduced. (ii) A specific interaction takes place between the macromolecule and the dye, thus increasing the local concentration of dye near the macromolecule. Actually, evidence was given by absorption spectroscopy for such an interaction between the anionic dendrimer and the diluted dye [22], while such an interaction was not detected with DNA for example. It can be noted that, according to the two hypotheses, an increase in dye supersaturation could take place, thus favouring aggregation. 


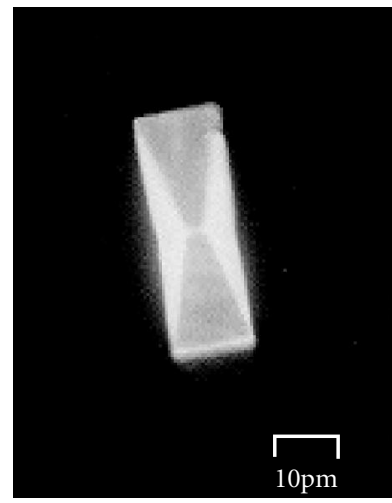

(a)

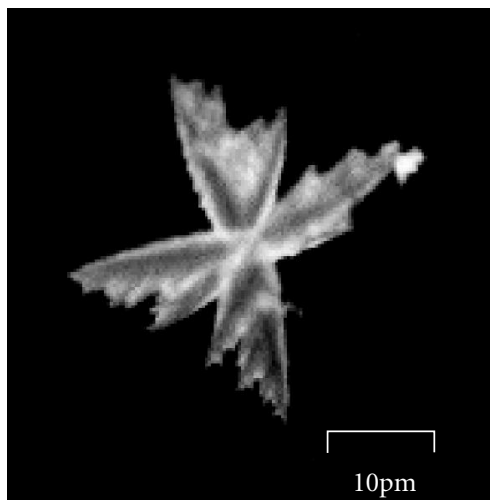

(b)

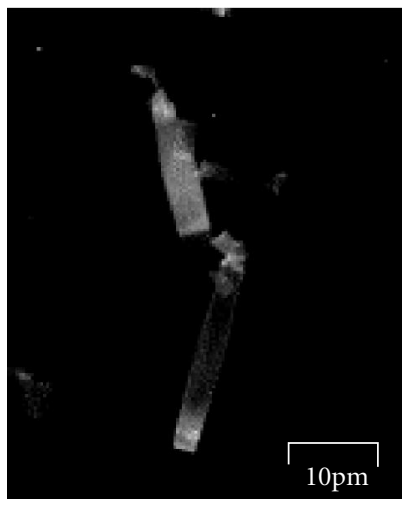

(c)

Figure 2: Fluorescence microscopy image of microcrystals of 1 grown in the presence of (a) poly(acrylic acid, sodium salt) $\left(5.9 \times 10^{-5} \mathrm{M}\right)$; (b) poly(acrylamide) $\left(4.1 \times 10^{-7} \mathrm{M}\right)$; and (c) calf thymus DNA $\left(5.0 \times 10^{-5} \mathrm{Mbp}\right)$. Total dye concentration: $2 \times 10^{-5} \mathrm{M}$.

The second and third steps of the recrystallization process are the formation of large aggregates and their evolution towards microcrystals. To understand the role that the macromolecule can play in these steps, it is interesting to investigate the nature of the microcrystals.

\subsection{Surface properties}

The question that arises is to know whether the microcrystals observed are made of pure dye or have some additive incorporated. Actually, microanalysis performed on microcrystals grown in the presence of G3.5 dendrimer [22] and poly(acrylic acid) [23] revealed that, after rinsing, the microcrystals contained a small amount of additive (17 and 7\% in weight, respectively). The surface electric zeta $(\zeta)$ potential, which refers to the charge acquired by a particle when it is placed in solution, was determined to get information about the surface properties. It was measured using a capillary electrophoresis technique based on the horizontal shift of the microparticles in an electric field. Although no reliable measurement could be obtained for the heavy agglomerates formed in water alone, the $\zeta$-potential was accurately determined for the five suspensions of microcrystals grown in the presence of additives. The results are collected in Table 1. It can be seen that the $\zeta$-potential was strongly negative with anionic dendrimer and polymers, almost neutral with poly(acrylamide) and positive with the cationic dendrimer. This suggests that part of the small amount of additive incorporated by the microcrystals is situated at the surface. Macromolecules can then act as a surface stabilizer, decreasing the interfacial tension and preventing the colloids from agglomerating. The fact that many functional groups are gathered on the same macromolecule makes the interaction quite strong.

\subsection{Observation of the microcrystals by fluorescence microscopy}

The macromolecules fixed on the microcrystal surface can lead to a shielding effect and interfere with crystal growth.
TABLE 1: Surface electric $\zeta$-potential measured for microcrystals grown in the presence of different additives.

\begin{tabular}{lcc}
\hline Additive & Functional group & $\zeta$-Potential $(\mathrm{mV})$ \\
\hline G3.5 dendrimer & $\mathrm{COO}^{-}$ & -48.6 \\
Poly(acrylic acid) & $\mathrm{COO}^{-}$ & -24.7 \\
Calf thymus DNA & $\mathrm{PO}_{4}^{-}$ & -22.1 \\
Poly(acrylamide) & $\mathrm{CONH}_{2}$ & -2.8 \\
G4 dendrimer & $\mathrm{NH}_{3}{ }^{+}$ & +15.4 \\
\hline
\end{tabular}

Consequently, a change in the microcrystal morphology can be expected. A drop of the microcrystal suspensions was deposited between slide and cover glass and observed under the fluorescence microscope, by exciting at 430-450 nm. All the microcrystals emitted above 500-530 nm. They were very flat, with a thickness in the nanometric range. The microcrystals formed in the presence of anionic dendrimer, cationic dendrimer, and poly(acrylic acid) appeared as rectangles, with a characteristic X-shape pattern at the centre (Figure $2 \mathrm{a}$ ). Their size varied from $40 \times 15$ to $4 \times 2^{-} \mathrm{m}$ for the two largest sides. The presence of poly(acrylamide) induced the formation of notched microcrystals $\left(34 \times 13^{-} \mathrm{m}\right)$, which sometimes assemble to give superstructures (Figure $2 \mathrm{~b}$ ). For DNA, two populations coexist. The first one consists in small rectangles $\left(4 \times 1^{-} \mathrm{m}\right)$ that emit yellow light, the second one is made of much longer rectangles (many of them measuring $20 \times 3^{-} \mathrm{m}$ ), which emit green light on their body and an intense orange-red light on their small extremities (Figure 2c).

\subsection{Optical properties}

The suspensions of free-standing microcrystals were easily studied by traditional spectroscopic methods. The UV/visible absorption spectrum of the suspensions is quite complex, with respect to the spectrum of the dissolved molecules. The latter only displays two bands: a band at short wavelength which corresponds to a $\pi-\pi^{*}$ transition, and a band at long wavelength, which is a charge transfer band. In water 


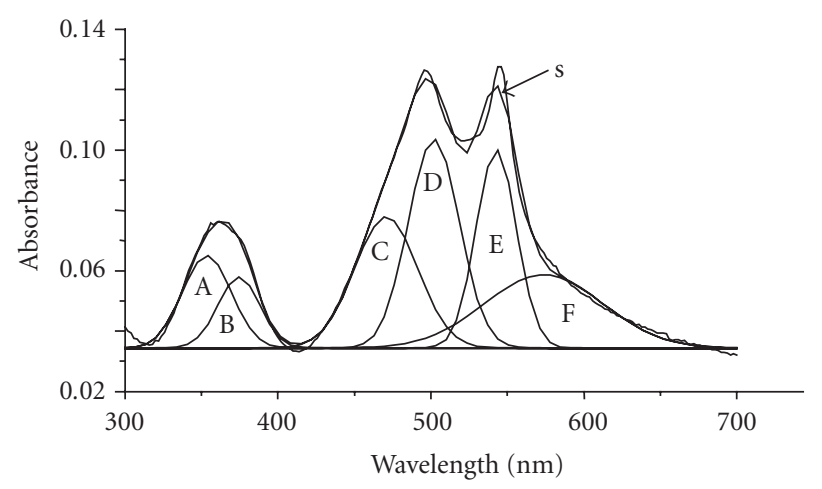

(a)

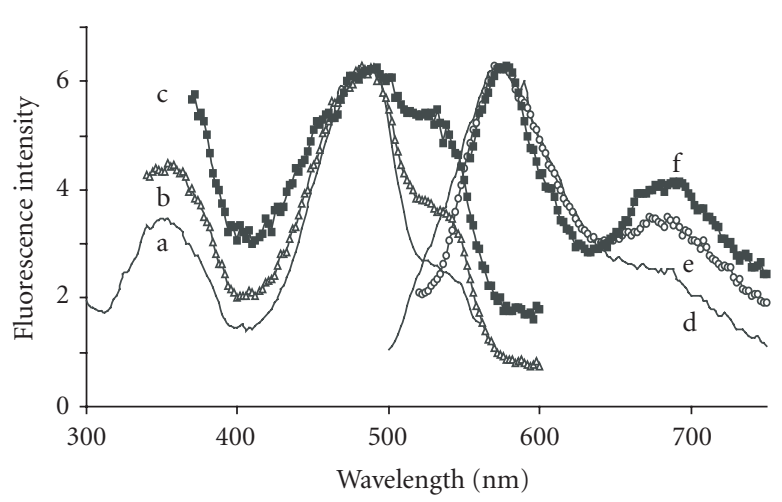

(b)

FIgURE 3: Top: UV/vis absorption spectrum of microcrystals of 1 grown in the presence of poly(acrylic acid, sodium salt) $(5.9 \times$ $10^{-4} \mathrm{M}$ ). Bands A to $\mathrm{F}$ were obtained by deconvolution, using six Gaussian functions. Spectrum s is the sum of the calculated bands. Bottom: Excitation spectrum (left) for different emission wavelengths (a) $570 \mathrm{~nm}$, (b) $660 \mathrm{~nm}$ and (c) $720 \mathrm{~nm}$; Emission spectrum (right) for different excitation wavelengths (d) $482 \mathrm{~nm}$, (e) 510 and (f) $545 \mathrm{~nm}$. Total dye concentration in the suspension: $2 \times 10^{-5} \mathrm{M}$.

with $2 \%$ ethanol these bands have a maximum at 346 and $482 \mathrm{~nm}$, respectively. For microcrystals, the absorption spectrum is actually composed of five [24] or six bands, which clearly appear after deconvolution, as illustrated on Figure 3, for the suspension obtained in the presence of poly(acrylic acid). The band maxima ranged from $353 \mathrm{~nm}$ to $578 \mathrm{~nm}$. It must be noted that the position of the bands hardly changed from one sample to another, but in contrast, their relative intensity underwent very strong variations. Most likely, the absorption spectrum arises from distinct populations of dye molecules, each experiencing a different environment. In a crystal unit cell, two adjacent molecules may be nonequivalent. It must not be forgotten either that in microcrystals, there is a high surface to volume ratio and a large number of defects. According to the location of the dye molecules in the microcrystal, the strength of the interaction with their neighbours may vary. The dye molecules may also be in contact with water molecules or not.

The emission behaviour is quite close for all the samples examined. For microcrystals grown in the presence of poly(acrylic acid, sodium salt), the emission spectrum, obtained by exciting at $482 \mathrm{~nm}$, displayed a maximum at $574 \mathrm{~nm}$. It was red-shifted with respect to the spectrum of the dissolved dye $\left(\lambda_{\max }=560 \mathrm{~nm}\right)$, and showed an additional small band at $684 \mathrm{~nm}$. The emission spectrum strongly depended on the excitation wavelength. When the excitation passed from 482 to $545 \mathrm{~nm}$, the main emission band got narrower, while the long-wavelength emission band became progressively more intense. When exciting above $570 \mathrm{~nm}$, emission was no longer detected, which shows that the bands situated in the red part of the absorption spectrum do not lead to emissive excited states. Conversely, the excitation spectrum varied with the emission wavelength. When emission was set at $570 \mathrm{~nm}$, the excitation spectrum displayed a strong band peaking at $484 \mathrm{~nm}$, similar to that of dissolved molecules, and a shoulder at $536 \mathrm{~nm}$. For high wavelength emissions, this shoulder became a band of strong intensity. It is clear that the microcrystal suspension contains several emitting species, some of them displaying the same spectral features as the dissolved dye molecules, while others exhibit totally different characteristics. Here again, stabilization depends on the interaction of the excited molecule with its crystalline surroundings. It can be recalled that the fluorescence quantum yield of the microcrystals grown in the presence of dendrimers and DNA was found to be close to $1 \times 10^{-3}$, by exciting at $482 \mathrm{~nm}$ [25]. By comparison, this value is one order of magnitude lower than that of an analogous 7 -amino-NBD dye in water [19].

\section{EXPERIMENTAL SECTION}

\subsection{Materials}

The fluorescent dye 4-n-octylamino-7-nitrobenz-2-oxa-1,3diazole (1) was synthesized as previously described [21]. Absolute ethanol was from Carlo Erba Reagenti. High-pressure demineralized water with a resistivity of $16 \mathrm{M} \Omega \mathrm{cm}$ was produced by a Milli-Q apparatus (Millipore). Anionic and cationic PAMAM starburst dendrimers, 3.5 and 4 generation, respectively, were purchased from Aldrich. Poly(acrylic acid, sodium salt) (average $\mathrm{MW}=5100 \mathrm{~g} \mathrm{~mol}^{-1}$ ) and poly(acrylamide) (average MW $=5 \times 10^{6}-6 \times 10^{6} \mathrm{~g} \mathrm{~mol}^{-1}$ ) also were from Aldrich. Calf thymus DNA, "highly polymerised" sodium salt, was from Sigma. The precise concentration of DNA in solutions was determined by UV/vis absorption spectroscopy using $\varepsilon^{260}=13200 \mathrm{M}^{-1} \mathrm{~cm}^{-1}$ for one base pair (bp) [26].

\subsection{Apparatus}

The size and shape of the microcrystals were observed with a Zeiss Axioskop fluorescence microscope, equipped with a standard camera. The objective and the eyepiece were provided with lenses of $\times 40$ or $\times 100$, and $\times 2.5$ magnification, respectively. The surface $\zeta$-potential was measured at room temperature using a Zetasizer $3000 \mathrm{HS}$ apparatus from Malvern Instruments, based on the Doppler effect. Spectroscopic measurements were carried out at $25^{\circ} \mathrm{C}$, in a thermostatted cell. UV/vis absorption spectra were recorded on 
a Hewlett-Packard 8452A diode array spectrophotometer. Corrected steady-state fluorescence spectra were registered on a Photon Technology International (PTI) Quanta Master 1 spectrofluorometer.

\section{CONCLUSION}

The reprecipitation method is a simple and versatile way to produce free-standing microcrystals. However, at the moment, it is not efficient enough to produce large amounts of microparticles, and still requires further developments to be adapted for industrial use. We have seen that changing the nature of the macromolecule used as additive allows the morphology of the microcrystals to be controlled, which leads to variations of their absorption and emission properties. This point is of particular interest for producing dye microcrystals in view of precise applications in optics. In a different area, biocompatible macromolecules, such as some polymers, can also be used for producing nano- and microparticles of pharmaceutical interest, thus improving the bioavailability and, as a result, the therapeutic efficiency of the drugs.

\section{ACKNOWLEDGMENT}

We are very grateful to NATO for financial support of Dr Liliana Bîrlă.

\section{REFERENCES}

[1] E. A. Silinsh, Organic Molecular Crystals: Their Electronic States, Springer, Berlin, Germany, 1980.

[2] L. Jinshui, W. Lun, G. Feng, L. Yongxing, and W. Yun, "Novel fluorescent colloids as a DNA fluorescence probe," Analytical and Bioanalytical Chemistry, vol. 377, no. 2, pp. 346-349, 2003.

[3] H. Y. Kim, T. G. Bjorklund, S.-H. Lim, and C. J. Bardeen, "Spectroscopic and photocatalytic properties of organic tetracene nanoparticles in aqueous solution," Langmuir, vol. 19, no. 9, pp. 3941-3946, 2003.

[4] H. Oikawa, H. Kasai, and H. Nakanishi, Anisotropic Organic Materials-Approaches to Polar Order, edited by R. Glaser and P. Kaszynski, ACS Symposium Series, no. 798, American Chemical Society, Washington, DC, USA, 2002.

[5] H. Masuhara, H. Nakanishi, and K. Sasaki, Eds., Single Organic Nanoparticles, Springer, Berlin, Germany, 2003.

[6] B. Yu. Shekunov and P. York, "Crystallization processes in pharmaceutical technology and drug delivery design," Journal of Crystal Growth, vol. 211, no. 1-4, pp. 122-136, 2000.

[7] N. Rasenack, H. Hartenhauer, and B. W. Müller, "Microcrystals for dissolution rate enhancement of poorly water-soluble drugs," International Journal of Pharmaceutics, vol. 254, no. 2, pp. 137-145, 2003.

[8] F. Debuigne, L. Jeunieau, M. Wiame, and J. B. Nagy, "Synthesis of organic nanoparticles in different W/O microemulsions," Langmuir, vol. 16, no. 20, pp. 7605-7611, 2000.

[9] A. H. Matsui, K. Mizuno, O. Nishi, et al., "Densities of states and band widths of excitons in anthracene microcrystallites embedded in PMMA," Chemical Physics, vol. 194, no. 1, pp. 167-174, 1995.

[10] N. Sanz, P. L. Baldeck, and A. Ibanez, "Organic nanocrystals embedded in sol-gel glasses for optical applications," Synthetic Metals, vol. 115, no. 1-3, pp. 229-234, 2000.
[11] A. Ibanez, S. Maximov, A. Guiu, C. Chaillout, and P. L. Baldeck, "Controlled nanocrystallization of organic molecules in sol-gel glasses," Advanced Materials, vol. 10, no. 18, pp. 1540-1543, 1999.

[12] H. Nakanishi and H. Katagi, "Microcrystals of polydiacetylene derivatives and their linear and nonlinear optical properties," Supramolecular Science, vol. 5, no. 3-4, pp. 289-295, 1998.

[13] K. Baba, H. Kasai, S. Okada, H. Oikawa, and H. Nakanishi, "Fabrication of organic nanocrystals using microwave irradiation and their optical properties," Optical Materials, vol. 21, no. 1-3, pp. 591-594, 2003.

[14] P. Kang, C. Chen, L. Hao, C. Zhu, Y. Hu, and Z. Chen, "A novel sonication route to prepare anthracene nanoparticles," Materials Research Bulletin, vol. 39, no. 4-5, pp. 545-551, 2004.

[15] D. Xiao, L. Xi, W. Yang, et al., "Size-tunable emission from 1,3-diphenyl-5-(2-anthryl)-2-pyrazoline nanoparticles," Journal of the American Chemical Society, vol. 125, no. 22, pp. 6740-6745, 2003.

[16] T. Oshikiri, H. Kasai, H. Katagi, S. Okada, H. Oikawa, and H. Nakanishi, "Fabrication of polydiacetylene fibrous microcrystals by the reprecipitation method," Molecular Crystals \& Liquid Crystals, vol. 337, pp. 25-30, 1999.

[17] T. Onodera, T. Oshikiri, H. Katagi, et al., "Nano-wire crystals of $\pi$-conjugated organic materials," Journal of Crystal Growth, vol. 229, no. 1-4, pp. 586-590, 2001.

[18] X. Ji, Y. Ma, Y. Cao, et al., "A study of the formation and embedding of perylene nanocrystals," Dyes and Pigments, vol. 51, no. 2, pp. 87-91, 2001.

[19] S. Fery-Forgues, J.-P. Fayet, and A. Lopez, "Drastic changes in the fluorescence properties of NBD probes with the polarity of the medium: involvement of a TICT state?" Journal of Photochemistry and Photobiology A: Chemistry, vol. 70, no. 3, pp. 229-243, 1993.

[20] A. Chattopadhyay, "Chemistry and biology of $N$-(7-nitrobenz-2-oxa-1,3-diazol-4-yl)-labeled lipids: fluorescent probes of biological and model membranes," Chemistry and Physics of Lipids, vol. 53, no. 1, pp. 1-15, 1990.

[21] F. Galinier, F. Bertorelle, and S. Fery-Forgues, "Spectrophotometric study of the incorporation of NBD probes in micelles: is a long alkyl chain on the fluorophore an advantage?" Comptes Rendus de l'Academie des Sciences Series IIC Chemistry, vol. 4, no. 12, pp. 941-950, 2001.

[22] F. Bertorelle, D. Lavabre, and S. Fery-Forgues, "Dendrimertuned formation of luminescent organic microcrystals," Journal of the American Chemical Society, vol. 125, no. 20, pp. 6244-6253, 2003.

[23] M. Abyan, F. Bertorelle, and S. Fery-Forgues, "Use of linear polymers to control the preparation of luminescent organic Microcrystals," Langmuir, vol. 21, no. 13, pp. 6030-6037, 2005.

[24] F. Bertorelle, F. Al-Ali, and S. Fery-Forgues, "Microcrystals of an organic fluorescent dye grown in the presence of various PAMAM dendrimers: control of the morphology and optical properties," International Journal of Photoenergy, vol. 6 , no. 4, pp. 221-225, 2004.

[25] F. Bertorelle and S. Fery-Forgues, "Morphology control of organic luminescent microcrystals grown in the presence of ionic and nonionic PAMAM dendrimers," Molecular Crystals \& Liquid Crystals, vol. 428, pp. 67-76, 2005.

[26] T. V. Chalikian, J. Völker, G. E. Plum, and K. J. Breslauer, "A more unified picture for the thermodynamics of nucleic acid duplex melting: a characterization by calorimetric and volumetric techniques," Proceedings of the National Academy of Sciences of the United States of America, vol. 96, no. 14, pp. 78537858, 1999. 


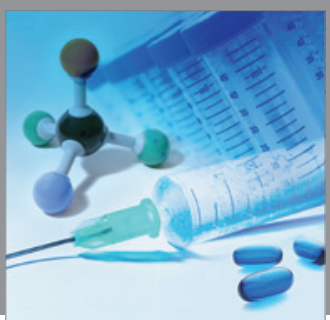

International Journal of

Medicinal Chemistry

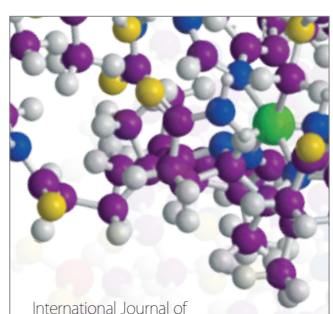

Carbohydrate Chemistry

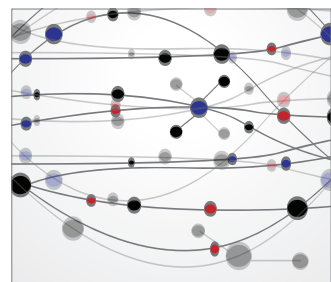

The Scientific World Journal
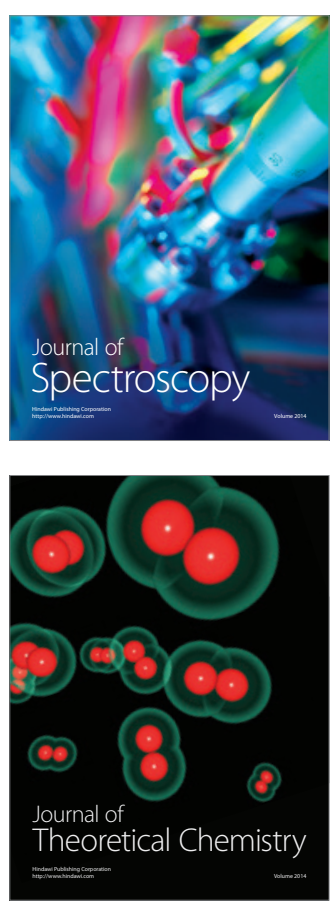
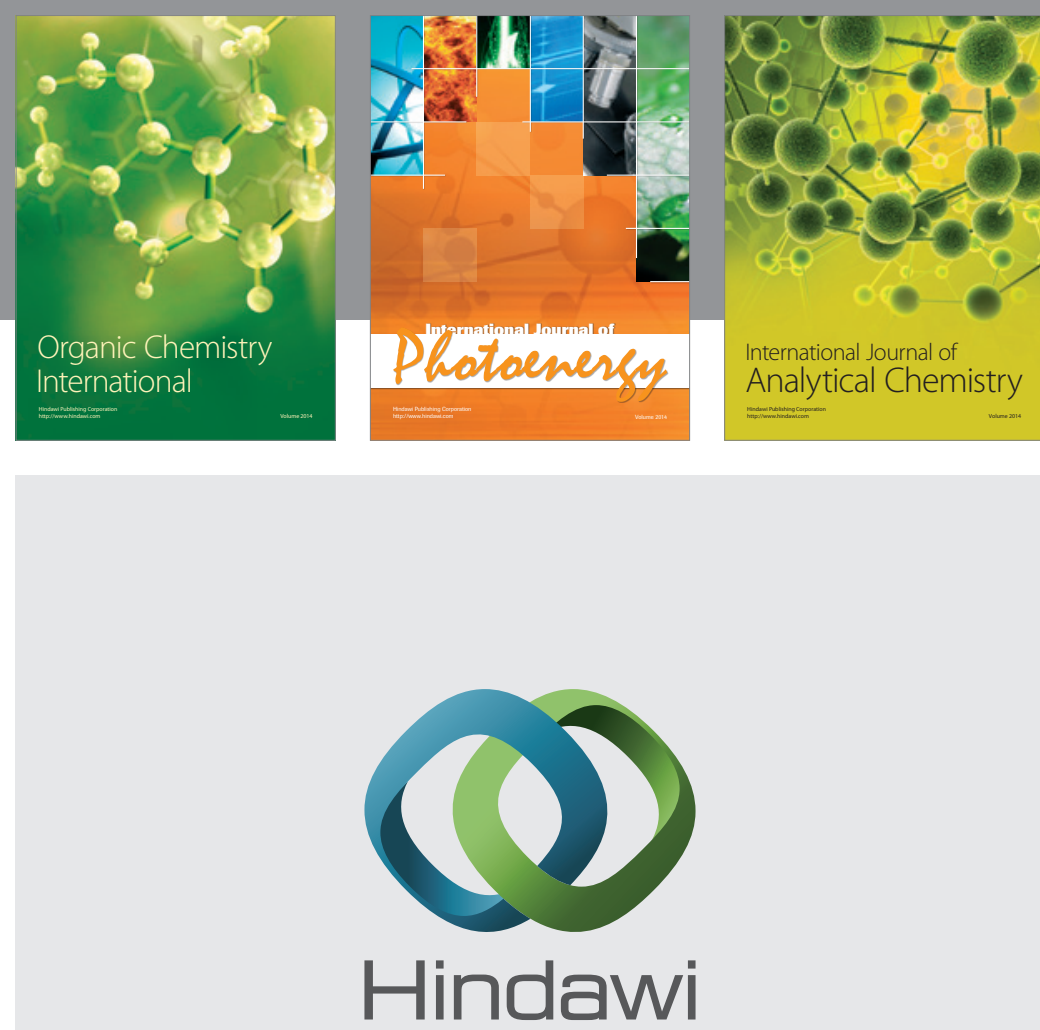

Submit your manuscripts at

http://www.hindawi.com
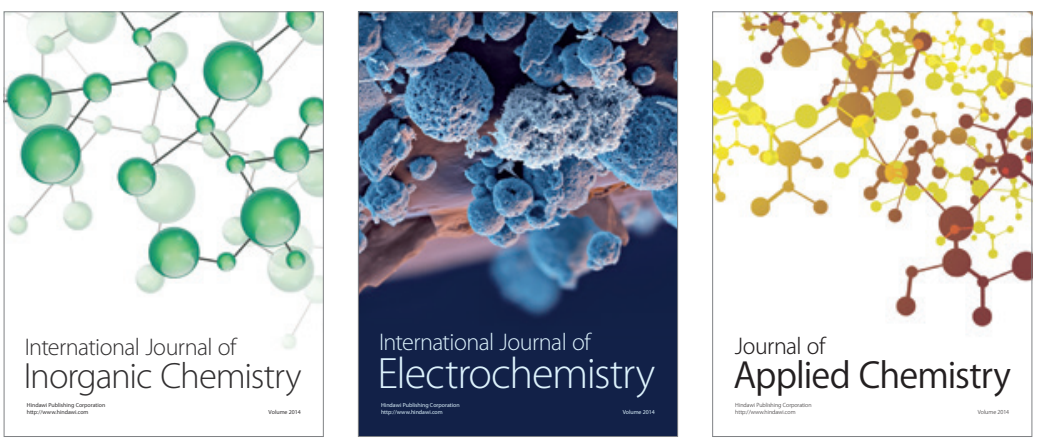

Journal of

Applied Chemistry
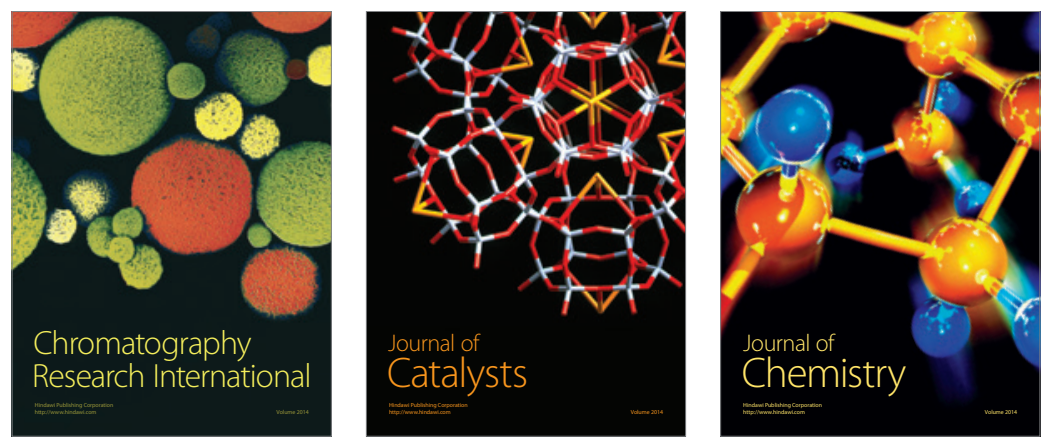
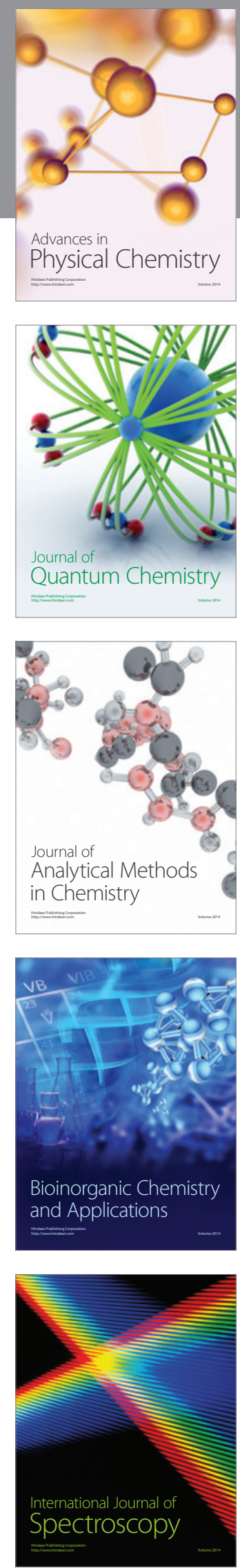